\title{
Sparsified Cholesky and Multigrid Solvers for Connection Laplacians *
}

\author{
Rasmus Kyng ${ }^{\dagger}$ \\ Yale University \\ New Haven CT, USA \\ rasmus.kyng@yale.edu
}

\author{
Yin Tat Lee ${ }^{\ddagger}$ \\ M.I.T. \\ Cambridge MA, USA \\ yintat@mit.edu
}

\author{
Richard Peng \\ Georgia Tech \\ Atlanta GA, USA \\ rpeng@cc.gatech.edu
}

\author{
Sushant Sachdeva \\ Yale University \\ New Haven CT, USA \\ sachdeva@cs.yale.edu
}

Daniel A. Spielman
Yale University
New Haven CT, USA
spielman@cs.yale.edu

\section{Categories and Subject Descriptors}

G.1.3 [Numerical Analysis]: Numerical Linear AlgebraSparse, structured, and very large systems (direct and iterative methods) gorithms accelerate Gaussian elimination by sparsifying the nonzero matrix entries created by the elimination process.

We use these new algorithms to derive the first nearly linear time algorithms for solving systems of equations in connection Laplacians - a generalization of Laplacian matrices that arise in many problems in image and signal processing.

We also prove that every connection Laplacian has a linear sized approximate inverse. This is an LU factorization with a linear number of nonzero entries that is a strong approximation of the original matrix. Using such a factorization one can solve systems of equations in a connection Laplacian in linear time. Such a factorization was unknown even for ordinary graph Laplacians.

\footnotetext{
*A full version of this paper is available on arXiv 12 . This paper incorporates and improves upon results previously announced by a subset of the authors in 13 .

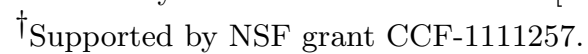

†Supported in part by NSF awards 0843915 and 1111109. Part of this work was done while visiting the Simons Institute for the Theory of Computing, UC Berkeley.

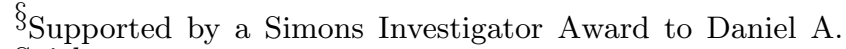
Spielman.

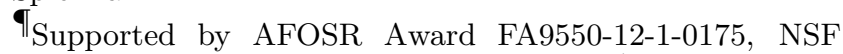
grant CCF-1111257, a Simons Investigator Award to Daniel A. Spielman, and a MacArthur Fellowship.
}

\footnotetext{
Permission to make digital or hard copies of all or part of this work for personal or classroom use is granted without fee provided that copies are not made or distributed for profit or commercial advantage and that copies bear this notice and the full citation on the first page. Copyrights for components of this work owned by others than ACM must be honored. Abstracting with credit is permitted. To copy otherwise, or republish, to post on servers or to redistribute to lists, requires prior specific permission and/or a fee. Request permissions from Permissions@acm.org.

Copyright is held by the owner/author(s). Publication rights licensed to ACM.

STOC'16, June 19-21, 2016, Cambridge, MA, USA

ACM. 978-1-4503-4132-5/16/06 \$ \$15.00

http://dx.doi.org/10.1145/2897518.2897640
}

\section{General Terms}

Algorithms, Theory

\section{Keywords}

Connection Laplacians, SDD linear systems, parallel algorithms, linear system solvers

\section{INTRODUCTION}

We introduce the sparsified Cholesky and sparsified multigrid algorithms for solving systems of linear equations. Two advantages of these algorithms over other recently introduced nearly-linear time algorithms for solving systems of equations in Laplacian matrices $26,24,9,10,8,5$, are:

1. They give nearly-linear time algorithms for solving systems of equations in a much broader class of matricesthe connection Laplacians and Hermitian block diagonally dominant matrices. Connection Laplacians 21. 3 are a generalization of graph Laplacians that arise in many applications, including celebrated work on cryoelectron microscopy [20, 19, 27, phase retrieval 1, 14, and many image processing problems (e.g. 17, 2]). Previous algorithms for solving systems of equations in graph Laplacians cannot be extended to solve equations in connection Laplacians because the previous algorithms relied on some form of low stretch spangeneral connection Laplacians.

2. They provide linear-sized approximate inverses of connection Laplacian matrices. That is, for every $n$-dimensional connection Laplacian $\boldsymbol{M}$, the sparsified Cholesky factorization algorithm produces an block-upper-triangular matrix $\boldsymbol{U}$ and block diagonal $\boldsymbol{D}$ with $O(n)$ nonzero entries so that $\boldsymbol{U}^{T} \boldsymbol{D} \boldsymbol{U}$ is a constant-factor approximation of $\boldsymbol{M}$. Such matrices $\boldsymbol{U}$ and $\boldsymbol{D}$ allow one to solve systems of equations in $\boldsymbol{M}$ to accuracy $\epsilon$ in time ning trees, a concept that has no analog for the more 
$O\left(m \log \epsilon^{-1}\right)$, where $m$ is the number of nonzero entries of $\boldsymbol{M}$. Even for ordinary Laplacian matrices, the existence of such approximate inverses is entirely new. The one caveat of this result is that we do not yet know how to compute those approximate inverses in nearly linear time.

The sparsified Cholesky and sparsified multigrid algorithms work by sparsifying the matrices produced during Gaussian elimination. Recall that Cholesky factorization is the version of Gaussian elimination for symmetric matrices, and that the high running time of Gaussian elimination comes from fillnew nonzero matrix entries that are created by row operations. If Gaussian elimination never produced rows with a super-constant number of entries, then it would run in linear time. The sparsified Cholesky algorithm accelerates Gaussian elimination by sparsifying the rows that are produced by elimination, thereby guaranteeing that the elimination will be fast. Sparsified Cholesky is inspired by one of the major advances in algorithms for solving linear equations in Laplacian matrices - the Incomplete Cholesky factorization (ICC) 15]. However, ICC merely drops entries produced by elimination, whereas sparsification also carefully increases ones that remain. The difference is crucial, and is why ICC does not provide a nearly-linear time solver.

To control the error introduced by sparsification, we have to be careful not to do it too often. This means that our algorithm actually chooses a large set of rows and columns to eliminate at once, and then sparsifies the result. This is the basis of our first algorithms, which establish the existence of linear time solvers and linear sized approximate inverses, after precomputation. This precomputation is analogous to the procedure of computing a matrix inverse: the approximate inverse takes much less time to apply than to compute.

To produce entire algorithms that run in nearly linear time (both to compute and apply the approximate inverse) requires a little more work. To avoid the work of computing the matrix obtained by eliminating the large set of rows and columns, we design a fast algorithm for approximating it quickly. This resulting algorithm produces a solver routine that does a little more work at each level, and so resembles a multigrid V-cycle 25. We call the resulting algorithm the sparsified multigrid. We note that Krishnan, Fattal, and Szeliski 11] present experimental results from the use of a sparsification heuristic in a multigrid algorithm for solving problems in computer vision.

Comparison to Peng-Spielman [18]. Our new algorithms are most closely related to the Laplacian solver recently introduced by Peng and Spielman [18: unlike previous Laplacian solvers, they rely only on sparsification and not on low stretch spanning trees, or other "support theory" based preconditioners. However, the sparsification in PengSpielman is still based on graph-theoretic algorithms 23 , 16. To sparsify without using graph theoretic algorithms, we employ a recently developed algorithm of Cohen et. al. 6] that allows us to sparsify a matrix by solving systems of equations in a subsampled matrix. This allows us to recursively solve a much broader family of linear systems.

There is a significant obstacle that prevents incorporating such a subsampling-based sparsification routine into an algorithm such as the one introduced by Peng and Spielman. Whereas previous algorithms for solving Laplacian systems produce sequences of matrices of smaller dimension, the Peng-Spielman algorithm constructs a sequence of matri- ces of the same dimension, with increasingly better condition numbers. It is the improvement in condition numbers that guarantees the convergence of the Peng-Spielman algorithm. Each of the matrices produced by the algorithm requires sparsification. However, sub-sampling produces matrices of worse condition number. Thus, we cannot sparsify the matrices produced by Peng-Spielman by recursively applying their solver on sub-sampled matrices.

Our new algorithm overcomes this difficulty by producing matrices of smaller dimension. The algorithm makes progress by eliminating variables rather than improving condition numbers. This also allows us to achieve sparsification by applying our algorithm recursively to subsampled matrices.

\subsection{Connection Laplacians and Block DD Ma- trices}

In this section, we define block diagonally dominant (bDD) matrices - the most general family of matrices such that the associated systems of linear equations can be solved by our algorithms. We begin by defining our motivating case: the connection Laplacians

Connection Laplacians may be thought of as a generalization of graph Laplacians where every vertex is associated with a vector, instead of a real number, and every edge is associated with a unitary matrix. Like graph Laplacians, they describe a natural quadratic form. Let $M_{[i, j]} \in \mathbb{C}^{r \times r}$ be the unitary matrix associated with edge $(i, j)$, and let $w_{i, j}$ be the (nonnegative) weight of edge $(i, j)$. We require that $\boldsymbol{M}_{[i, j]}=\boldsymbol{M}_{[j, i]}^{*}$, where $*$ denotes the conjugate transpose. The quadratic form associated with this connection Laplacian is a function of vectors $\boldsymbol{v}^{(i)} \in \mathbb{C}^{r}$, one for each vertex $i$, that equals

$$
\sum_{(i, j) \in E} \boldsymbol{w}_{i, j}\left\|\boldsymbol{v}^{(i)}-\boldsymbol{M}_{[i, j]} \boldsymbol{v}^{(j)}\right\|^{2}
$$

The matrix corresponding to this quadratic form is a block matrix with blocks $\boldsymbol{M}_{[i, j]}$. Most applications of the connection Laplacian require one to either solve systems of linear equations in this matrix, or to compute approximations of its smallest eigenvalues and eigenvectors. By applying the inverse power method (or inverse Lanczos), we can perform these eigenvector calculations by solving a logarithmic number of linear systems in the matrix (see [24, Section 7]).

The matrices obtained from the connection Laplacian are a special case of block diagonally dominant (bDD) matrices, which we now define. Throughout this paper, we consider block matrices having entries in $\mathbb{C}^{r \times r}$, where $r>0$ is a fixed integer. We say that $\boldsymbol{M} \in\left(\mathbb{C}^{r \times r}\right)^{m \times n}$ has $m$ block-rows, and $n$ block-columns. For $i \in[m], j \in[n]$, we let $\boldsymbol{M}_{[i, j]} \in$ $\mathbb{C}^{r \times r}$ denote the $i, j$-block in $\boldsymbol{M}$, and $\boldsymbol{M}_{[j]}$ denote the $j^{\text {th }}$ block-column, i.e., $\boldsymbol{M}_{[j]}=\left[\left(\boldsymbol{M}_{[1, j]}\right)^{*},\left(\boldsymbol{M}_{[2, j]}\right)^{*}, \ldots,\left(\boldsymbol{M}_{[m, j]}\right)^{*}\right]^{*}$. For sets $F \subseteq[m], C \subseteq[n]$, we let $M_{[F, C]} \in\left(\mathbb{C}^{r \times r}\right)^{|F| \times|C|}$ denote the block-submatrix with blocks $\boldsymbol{M}_{[i, j]}$ for $i \in F, j \in C$. $\boldsymbol{M}$ is block-diagonal, if $\boldsymbol{M}_{[i, j]}=0$ for $i \neq j$. We emphasize that all computations are done over $\mathbb{C}$, not over a matrix group.

To define bDD matrices, let $\|\boldsymbol{A}\|$ denote the operator norm ${ }^{1}$ of a matrix $\boldsymbol{A}$. For Hermitian matrices $\boldsymbol{A}, \boldsymbol{B}$, write $\boldsymbol{A} \succeq \boldsymbol{B}$ iff $\boldsymbol{A}-\boldsymbol{B}$ is positive semidefinite.

\footnotetext{
${ }^{1}$ Recall that the operator norm is the largest singular value of $\boldsymbol{A}$ and square root of the largest eigenvalue of $\boldsymbol{A}^{*} \boldsymbol{A}$.
} 
Definition 1. A Hermitian block-matrix $M \in\left(\mathbb{C}^{r \times r}\right)^{n \times n}$ is block diagonally dominant (or bDD) if

$$
\text { for all } i \in[n], \quad M_{[i, i]} \succeq \mathbb{I}_{r} \cdot \sum_{j: j \neq i}\left\|M_{[i, j]}\right\|,
$$

where $\mathbb{I}_{r} \in \mathbb{C}^{r \times r}$ denotes the identity matrix.

Equivalently, a Hermitian $\boldsymbol{M}$ is a bDD matrix iff it can be written as $\boldsymbol{D}-\boldsymbol{A}$ where $\boldsymbol{D}$ is block-diagonal, and $\boldsymbol{D}_{[i, i]} \succeq$ $\mathbb{I}_{r} \sum_{j}\left\|\boldsymbol{A}_{[i, j]}\right\|$ (see full version for a proof 12 , Lemma B.1]). Throughout the paper we treat $r$ as a constant. The hidden dependence on $r$, of the running times of the algorithms we present, is polynomial. There are two key properties of bDD matrices that allow us to solve linear systems in these matrices fast. Firstly, bDD matrices are closed under Schur complement. Secondly, bDD matrices can quickly be decomposed into an explicit sum of bDD matrices where each term has small support. This is essentially enough to ensure that nearly linear time solvers exist, but not enough to give a fast algorithm for constructing such a solver. To construct a fast solver, we need to efficiently construct a sparse approximation to the Schur complement of a bDD matrix.

The major results of this paper are the following.

Theorem 1 (Sparsified Multigrid). There is an algorithm that, when given a bDD matrix $\boldsymbol{M}$ with $n$ block rows and $m$ nonzero blocks, produces a solver for $\boldsymbol{M}$ in $O\left(m \log n+n \log ^{2+o(1)} n\right)$ work and $O\left(n^{o(1)}\right)$ depth such that the solver can compute $\epsilon$-approximate solutions to systems of equations in $\boldsymbol{M}$ in $O\left(\left(m+n \log ^{1+o(1)} n\right) \log (1 / \epsilon)\right)$ work and $O\left(\log ^{2+o(1)} n \log (1 / \epsilon)\right)$ depth.

Previously, the existence of nearly-linear time solvers was even unknown for the 1-dimensional case $(r=1)$ when the off-diagonal entries were allowed to be complex numbers.

We can use the above algorithm to find approximations of the smallest eigenvalues and eigenvectors of such matrices at an additional logarithmic cost.

Theorem 2 (Sparsified Cholesky). For every $b D D$ matrix $\boldsymbol{M}$ with $n$ block-rows there exists a diagonal matrix $\boldsymbol{D}$ and an upper triangular matrix $\boldsymbol{U}$ with $O(n)$ nonzero blocks so that

$$
\boldsymbol{U}^{T} \boldsymbol{D} \boldsymbol{U} \approx_{3 / 4} \boldsymbol{M}
$$

Moreover, linear equations in $\boldsymbol{U}, \boldsymbol{U}^{T}$, and $\boldsymbol{D}$ can be solved with linear work in depth $O\left(\log ^{2} n\right)$, and these matrices can be computed in polynomial time.

These matrices allow one to solve systems of linear equations in $M$ to $\epsilon$-accuracy in parallel time $O\left(\log ^{2} n \log ^{-1} \epsilon\right)$ and work $O\left(m \log ^{-1} \epsilon\right)$. Results of this form were previously unknown even for graph Laplacians.

In the next two sections we explain the ideas used to prove these theorems. Proofs may be found in the appendices that follow.

\section{BACKGROUND}

We require some standard facts about the $\preccurlyeq$ order on matrices.

Fact 1. For $\boldsymbol{A}$ and $\boldsymbol{B}$ positive definite, $\boldsymbol{A} \succcurlyeq \boldsymbol{B}$ if and only if $\boldsymbol{B}^{-1} \succcurlyeq \boldsymbol{A}^{-1}$.

Fact 2. If $\boldsymbol{A} \succcurlyeq \boldsymbol{B}$ and $\boldsymbol{C}$ is any matrix of compatible dimension, then $\boldsymbol{C} \boldsymbol{A} \boldsymbol{C}^{T} \succcurlyeq \boldsymbol{C B} \boldsymbol{C}^{T}$.
We say that $\boldsymbol{A}$ is an $\epsilon$-approximation of $\boldsymbol{B}$, written $\boldsymbol{A} \approx_{\epsilon} \boldsymbol{B}$, if $e^{\epsilon} \boldsymbol{B} \succcurlyeq \boldsymbol{A} \succcurlyeq e^{-\epsilon} \boldsymbol{B}$. This relation is symmetric. We say that $\tilde{\boldsymbol{x}}$ is an $\epsilon$-approximate solution to the system $\boldsymbol{A} \boldsymbol{x}=\boldsymbol{b}$ if $\left\|\tilde{\boldsymbol{x}}-\boldsymbol{A}^{-1} \boldsymbol{b}\right\|_{\boldsymbol{A}} \leq \epsilon\|\boldsymbol{x}\|_{\boldsymbol{A}}$, where $\|\boldsymbol{x}\|_{\boldsymbol{A}}=\left(\boldsymbol{x}^{T} \boldsymbol{A} \boldsymbol{x}\right)^{1 / 2}$. If $\epsilon<1 / 2, \boldsymbol{A} \approx_{\epsilon} \boldsymbol{B}$ and $\boldsymbol{B} \tilde{\boldsymbol{x}}=\boldsymbol{b}$, then $\tilde{\boldsymbol{x}}$ is a $2 \epsilon$ approximate solution to $\boldsymbol{A} \boldsymbol{x}=\boldsymbol{b}$.

Fact 3. If $\boldsymbol{A} \approx_{\epsilon} \boldsymbol{B}$ and $\boldsymbol{B} \approx_{\delta} \boldsymbol{C}$, then $\boldsymbol{A} \approx_{\epsilon+\delta} \boldsymbol{C}$.

We now address one technicality of dealing with $b D D$ matrices: it is not immediate whether or not a $b D D$ matrix is singular. Moreover, if it is singular, the structure of its null space is not immediately clear either. Throughout the rest of this paper, we will consider $b D D$ matrices to which a small multiple of the identity have been added. These matrices will be nonsingular. To reduce the problem of solving equations in a general $b D D$ matrix $\boldsymbol{M}$ to that of solving equations in a nonsingular matrix, we require an estimate of the smallest nonzero eigenvalue of $\boldsymbol{M}$.

Claim 1. Suppose that all nonzero eigenvalues of $M$ are at least $\mu$ and $\boldsymbol{Z} \approx_{\epsilon}(M+\epsilon \mu \mathbb{I})^{-1}$ for some $0<\epsilon<1 / 2$. Given any $\boldsymbol{b}$ such that $\boldsymbol{M} \boldsymbol{x}=\boldsymbol{b}$ for some $\boldsymbol{x}$, we have

$$
\|\boldsymbol{x}-\boldsymbol{Z} \boldsymbol{b}\|_{M}^{2} \leq 6 \epsilon\|x\|_{M}^{2} .
$$

Hence, we can solve systems in $\boldsymbol{M}$ by approximately solving systems in $\boldsymbol{M}+\epsilon \mu \boldsymbol{I}$. Any lower bound $\mu$ on the smallest nonzero eigenvalue of $\boldsymbol{M}$ will suffice. It only impacts the running times of our algorithms in the numerical precision with which one must carry out the computations.

The above claim allows us to solve systems in $\boldsymbol{M}$ that have a solution. If $\boldsymbol{M}$ is singular and we want to apply its pseudoinverse (that is, to find the closest solution in the range of $\boldsymbol{M}$ ), we can do so by pre and post multiplying by $\boldsymbol{M}$ to project onto its range. The resulting algorithm, which is implicit in the following claim, requires applying a solver for $M$ three times. It also takes $O(\log \kappa)$ times as long to run, where $\kappa$ is the finite condition number ${ }^{2}$ of $\boldsymbol{M}$.

Claim 2. Let $\kappa$ be an upper bound on the finite condition number of $\boldsymbol{M}$. Given an error parameter $0<\epsilon<1$, let $\delta=\epsilon / 56 \kappa^{3}$. If all nonzero eigenvalues of $M$ are at least $\mu$, and if $\boldsymbol{Z} \approx_{\delta}(\boldsymbol{M}+\epsilon \mu \boldsymbol{I})^{-1}$, then

$$
M Z^{3} M \approx_{4 \epsilon} M^{+} .
$$

\section{OVERVIEW OF THE ALGORITHMS}

We index the block rows and columns of a block matrix by a set of vertices (or indices) $V$. When we perform an elimination, we eliminate a set $F \subset V$, and let $C=V-$ $F$. Here, $F$ stands for "fine" and $C$ stands for "coarse". In contrast with standard multigrid methods, we will have $|F| \leq|C|$.

To describe block-Cholesky factorization, we write the matrix $\boldsymbol{M}$ with the rows and columns in $F$ first:

$$
\boldsymbol{M}=\left[\begin{array}{ll}
\boldsymbol{M}_{[F, F]} & \boldsymbol{M}_{[F, C]} \\
\boldsymbol{M}_{[C, F]} & \boldsymbol{M}_{[C, C]}
\end{array}\right] .
$$

Cholesky factorization writes the inverse of this matrix as

$$
\begin{gathered}
\left.\boldsymbol{M}^{-1}=\left[\begin{array}{cc}
\boldsymbol{I} & \left.-\boldsymbol{M}_{[F, F]}^{-1} \boldsymbol{M}_{[F, C]}\right] \\
0 & \boldsymbol{I}
\end{array}\right] \begin{array}{cc}
\boldsymbol{M}_{[F, F]}^{-1} & 0 \\
0 & S c(\boldsymbol{M}, F)^{-1}
\end{array}\right] \\
{\left[\begin{array}{cc}
\boldsymbol{I} & 0 \\
-\boldsymbol{M}_{[C, F]} \boldsymbol{M}_{[F, F]}^{-1} & \boldsymbol{I}
\end{array}\right],}
\end{gathered}
$$

${ }^{2}$ The finite condition number is the ratio of the largest singular value to the smallest nonzero singular value. 
where

$$
S c(\boldsymbol{M}, F) \stackrel{\text { def }}{=} \boldsymbol{M}_{[C, C]}-\boldsymbol{M}_{[C, F]} \boldsymbol{M}_{[F, F]}^{-1} \boldsymbol{M}_{[F, C]}
$$

is the Schur complement of $M$ with respect to $F$.

Our algorithms rely on two fundamental facts about $b D D$ matrices: that the Schur complement of a $b D D$ matrix is a $b D D$ matrix (see full version for a proof [12, Lemma B.3]) and that one can sparsify $b D D$ matrices. The following theorem is implicit in 7 .

TheOREM 3. For every $\epsilon \leq 1$, every bDD matrix $\boldsymbol{M}$ with $n$ r-dimensional block rows and columns can be $\epsilon$-approximated by a bDD matrix having at most $10 \mathrm{nr} / \epsilon^{2}$ nonzero blocks.

We use the identity (1) to reduce the problem of solving a system of equations in $\boldsymbol{M}$ to that of solving equations in its Schur complement. The easiest part of this is multiplication by $M_{[C, F]}$ : the time is proportional to the number of nonzero entries in the submatrix, and we can sparsify $\boldsymbol{M}$ to guarantee that this is small.

The costlier part of the reduction is the application of the inverse of $\boldsymbol{M}_{[F, F]}$ three times. This would be fast if $\boldsymbol{M}_{[F, F]}$ were block-diagonal, which corresponds to $F$ being an independent set. We cannot find a sufficiently large independent set $F$, but we can find a large set that is almost independent. This results in a matrix $\boldsymbol{M}_{[F, F]}$ that is well approximated by its diagonal, and thus linear equations in this matrix can be quickly solved to high accuracy by a few Jacobi iterations (see Theorem 4).

We can prove the existence of linear sized approximate inverses by explicitly computing $S c(\boldsymbol{M}, F)$, sparsifying it, and then recursively applying the algorithm just described. To make this algorithm efficient, we must compute a sparse approximation to $S c(\boldsymbol{M}, F)$ without constructing $S c(\boldsymbol{M}, F)$. This is the problem of spectral vertex sparsification, and we provide a fast algorithm for this task in Sections 3.4

\subsection{Schur Complement Chains}

We encode this recursive algorithm by a Schur complement chain (SCC). An SCC defines a linear operator that can be used to approximately solve equations in an initial matrix $\boldsymbol{M}^{(0)}$. If the $b D D$ matrix $\boldsymbol{M}$ is sparse, then it is the same as $\boldsymbol{M}^{(0)}$; if not, then $\boldsymbol{M}^{(0)}$ is a sparse approximation to $\boldsymbol{M}$. Let $F_{1}$ be the first set of vertices eliminated, $\boldsymbol{M}^{(1)}$ a sparse approximation to the Schur complement of $\boldsymbol{M}^{(0)}$ with respect to $F_{1}$, and $Z^{(1)}$ an operators that approximates the inverse of $\boldsymbol{M}_{\left[F_{1}, F_{1}\right]}^{(0)}$.

Definition 2. (Schur Complement Chain) An $\boldsymbol{\epsilon}$-Schur complement chain $\left(\boldsymbol{\epsilon}\right.$-SCC) for a matrix $\boldsymbol{M}^{0}$ indexed by vertex set $V$ is a sequence of operators and subsets,

$$
\left(\left(\boldsymbol{M}^{(1)}, \boldsymbol{Z}^{(1)}\right), \ldots,\left(\boldsymbol{M}^{(d)}, \boldsymbol{Z}^{(d)}\right) ; F_{1}, \ldots, F_{d}\right),
$$

so that for $C_{0}=V$ and $C_{i+1}=C_{i} \backslash F_{i+1},\left|C_{d}\right| \leq 1000$ and for $1 \leq i \leq d$, we have $\boldsymbol{M}^{(i)} \approx_{\epsilon_{i}} S c\left(\boldsymbol{M}^{(i-1)}, F_{i}\right)$, and

$$
0 \preceq\left(\boldsymbol{Z}^{(i)}\right)^{-1}-\boldsymbol{M}_{\left[F_{i}, F_{i}\right]}^{(i-1)} \preceq \epsilon_{i} \cdot S c\left(\boldsymbol{M}^{(i-1)}, C_{i}\right) .
$$

The algorithm ApplyChain, described in Figure 1 applies an SCC to solve equations in $\boldsymbol{M}^{(0)}$ in the natural way and satisfies the following guarantee.

$$
\begin{gathered}
\boldsymbol{x}^{(0)}=\operatorname{ApplyChain}( \\
\left(\boldsymbol{M}^{(1)}, \boldsymbol{Z}^{(1)}\right), \ldots,\left(\boldsymbol{M}^{(d)}, \boldsymbol{Z}^{(d)}\right) ; \\
\left.F_{1}, \ldots, F_{d}\right)
\end{gathered}
$$

1. Initialize $\boldsymbol{b}^{(0)} \leftarrow \boldsymbol{b}$.

2. For $\mathrm{i}=1, \ldots, d$

(a) $\boldsymbol{x}_{\left[F_{i}\right]}^{(i-1)} \leftarrow \boldsymbol{Z}^{(i)} \boldsymbol{b}_{\left[F_{i}\right]}^{(i-1)}$,

(b) $\boldsymbol{b}^{(i)} \leftarrow \boldsymbol{b}_{\left[C_{i}\right]}^{(i-1)}-\boldsymbol{M}_{\left[C_{i}, F_{i}\right]}^{(i-1)} \boldsymbol{x}_{\left[F_{i}\right]}^{(i-1)}$.

3. $\boldsymbol{x}^{(d)} \leftarrow\left(\boldsymbol{M}^{(d)}\right)^{-1} \boldsymbol{b}^{(d)}$.

4. For $\mathrm{i}=d, \ldots, 1$

(a) $\boldsymbol{x}_{\left[C_{i}\right]}^{(i-1)} \leftarrow \boldsymbol{x}^{(i)}$.

(b) $\boldsymbol{x}_{\left[F_{i}\right]}^{(i-1)} \leftarrow \boldsymbol{x}_{\left[F_{i}\right]}^{(i-1)}-\boldsymbol{Z}^{(i)} \boldsymbol{M}_{\left[F_{i}, C_{i}\right]}^{(i-1)} \boldsymbol{x}^{(i)}$.

5. Return $\boldsymbol{x}^{(0)}$.

Figure 1: Solver Algorithm using Vertex Sparsifier Chain

LEMma 1. Consider an $\boldsymbol{\epsilon}-S C C$ for $\boldsymbol{M}^{(0)}$ where $\boldsymbol{M}^{(i)}$ and $Z^{(i)}$ can be applied to a vector in work $W_{M^{(i)}}, W_{D^{(i)}}$ and depth $D_{M^{(i)}}, D_{Z^{(i)}}$ respectively. The algorithm

$$
\operatorname{ApplyChain}\left(\left(\boldsymbol{M}^{(1)}, \boldsymbol{Z}^{(1)}\right), \ldots,\left(\boldsymbol{M}^{(d)}, \boldsymbol{Z}^{(d)}\right) ; F_{1}, \ldots, F_{d} ; \boldsymbol{b}\right)
$$

corresponds to a linear operator $\boldsymbol{W}$ acting on $\boldsymbol{b}$ such that

1. $\boldsymbol{W}^{-1} \approx_{\sum_{i=1}^{d} 2 \epsilon_{i}} \boldsymbol{M}^{(0)}$, and

2. for any vector $\boldsymbol{b}$, it runs in

$$
\begin{aligned}
& O\left(\sum_{i=1}^{d}\left(D_{M^{(i)}}+D_{Z^{(i)}}\right)\right) \text { depth and } \\
& O\left(\sum_{i=1}^{d}\left(W_{M^{(i)}}+W_{Z^{(i)}}\right)\right) \text { total work. }
\end{aligned}
$$

For an $\boldsymbol{\epsilon}$-SCC chain for $\boldsymbol{M}^{(0)}$, we define the depth and work of the chain to be the work and depth required by APPLYCHAIN to apply the SCC. A proof of the above Lemma is given in the full version [12, Section C].

\subsection{Choosing $F_{i}$ : $\alpha$-bDD Matrices}

We must choose the set of vertices $F_{i}$ so that we can approximate the inverse of $\boldsymbol{M}_{\left[F_{i}, F_{i}\right]}^{(i)}$ by an operator $\boldsymbol{Z}^{(i)}$ that is efficiently computable. We do this by requiring that the matrix $\boldsymbol{M}_{\left[F_{i}, F_{i}\right]}^{(i)}$ be $\alpha$-block diagonally dominant ( $\alpha$-bDD), a term that we now define.

Definition 3. A Hermitian block-matrix $\boldsymbol{M}$ is $\alpha$-bDD if

$$
\forall i, \quad \boldsymbol{M}_{[i, i]} \succcurlyeq(1+\alpha) \mathbb{I}_{r} \cdot \sum_{j: j \neq i}\left\|\boldsymbol{M}_{[i, j]}\right\| .
$$

We remark that a 0 -bDD matrix is simply a bDD matrix. In particular, for $r=1$, Laplacian matrices are 0-bDD.

By picking a subset of rows at random and discarding those that violate condition (2), the algorithm BDDSUBSET (described in Figure 2 finds a linear sized subset $F$ of the block-rows of a bDD matrix $\boldsymbol{M}$ so that $\boldsymbol{M}_{[F, F]}$ is $\alpha$-bDD. The following lemma describes the guarantee of BDDSUBSET. A complete proof is given in the full version 12 , Section D]. 


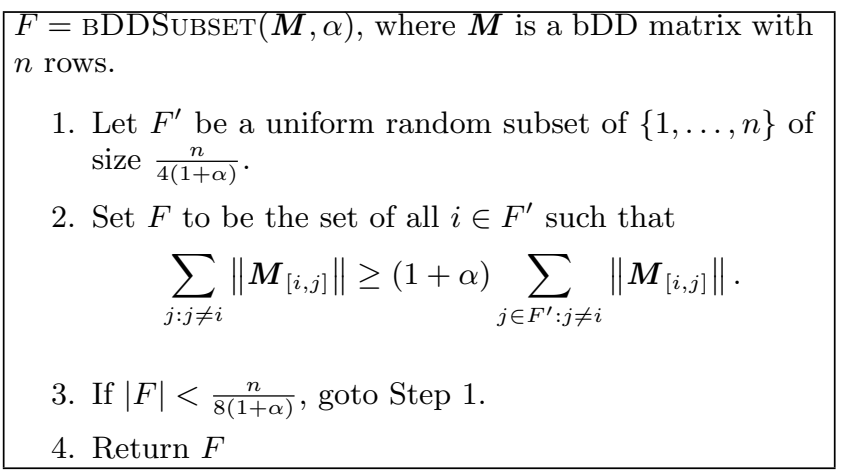

Figure 2: Routine for finding an $\alpha$-strongly block diagonally dominant submatrix

$\boldsymbol{x}=$ J ACOBI $(\epsilon, \boldsymbol{M}, \boldsymbol{b})$
1. Create the matrix $\boldsymbol{L}$ where
\[ \boldsymbol{L}_{[i, j]}=\left\{\begin{array}{l}\mathbb{I}_{r} \cdot \sum_{j: j \neq i}\left\|M_{[i, j]}\right\| \\ \boldsymbol{M}_{[i, j]}\end{array}\right. \]
2. Set $\boldsymbol{X}=\boldsymbol{M}-\boldsymbol{L}$.
3. Set $k$ to be an odd integer that is greater than
$\quad \log (3 / \epsilon)$.
4. Set $\boldsymbol{x}^{(0)}=\boldsymbol{X}^{-1} \boldsymbol{b}$.
5. For $i=1 \ldots k$ do
$\quad$ (a) Set $\boldsymbol{x}^{(i)}=-\boldsymbol{X}^{-1} \boldsymbol{L} \boldsymbol{x}^{(i-1)}+\boldsymbol{X}^{-1} \boldsymbol{b}$.
6. Return $\boldsymbol{x}^{(k)}$

Figure 3: Jacobi Iteration for Solving Linear Systems in an $\alpha$-bDD Matrix

Lemma 2. Given a bDD matrix $\boldsymbol{M}$ with $n$ block-rows, and an $\alpha \geq 0$, BDDSUBSET computes a subset $F$ of size least $n /(8(1+\alpha))$ such that $\boldsymbol{M}_{[F, F]}$ is $\alpha-b D D$. It runs in runs in $O(m)$ expected work and $O(\log n)$ expected depth, where $m$ is the number of nonzero blocks in $M$.

We can express an $\alpha$-bDD matrix as a sum of a block diagonal matrix and a bDD matrix so that it is well-approximated by the diagonal.

Lemma 3. Every $\alpha-b D D$ matrix $\boldsymbol{M}$ can be written in the form $\boldsymbol{X}+\boldsymbol{L}$ where $\boldsymbol{X}$ is block-diagonal, $\boldsymbol{L}$ is bDD, and $\boldsymbol{X} \succcurlyeq$ $\frac{\alpha}{2} \boldsymbol{L}$.

As $\boldsymbol{L}$ is positive semidefinite, $(1+2 / \alpha) \boldsymbol{X} \succcurlyeq \boldsymbol{M} \succcurlyeq \boldsymbol{X}$, which means that $\boldsymbol{X}$ is a good approximation of $\boldsymbol{M}$ when $\alpha$ is reasonably big. As block-diagonal matrices like $\boldsymbol{X}$ are easy to invert, systems in these well-conditioned matrices can be solved rapidly using preconditioned iterative methods. The following theorem proves that a variant of Jacobi iteration (described in Figure 3 provides an operator that satisfies the requirements of Definition 2, A complete proof is included in the full version 12 , Section E].

TheOREM 4. Let $\boldsymbol{M}$ be a bDD matrix with index set $V$, and let $F \subseteq V$ such that $M_{[F, F]}$ is $\alpha$-bDD for some $\alpha \geq 4$, and has $m_{F F}$ nonzero blocks. The algorithm

$\operatorname{JACOBI}\left(\epsilon, \boldsymbol{M}_{[F, F]}, \boldsymbol{b}\right)$ acts as a linear operator $\boldsymbol{Z}$ on $\boldsymbol{b}$ that satisfies

$$
0 \preceq(\boldsymbol{Z})^{-1}-\boldsymbol{M}_{[F, F]} \preceq \epsilon \cdot S c(\boldsymbol{M}, V \backslash F) .
$$

The algorithm takes $O\left(m_{F F} \log \left(\frac{1}{\epsilon}\right)\right)$ work and $O\left(\log n \log \left(\frac{1}{\epsilon}\right)\right)$ depth.

We now explain how Theorems 4 and 3 allow us to construct Schur complement chains that can be applied in nearly linear time. We optimize the construction in the next section.

Theorem 3 tells us that there is a $b D D$ matrix $\boldsymbol{M}^{(0)}$ with $O\left(n / \epsilon^{2}\right)$ nonzero blocks that that $\epsilon$-approximates $\boldsymbol{M}$, and that for every $i$ there is a $b D D$ matrix $\boldsymbol{M}^{(i+1)}$ with $O\left(\left|C_{i}\right| / \epsilon^{2}\right)$ nonzero blocks that is an $\epsilon$-approximation of $S c\left(M^{(i)}, F_{i}\right)$. We will pick $\epsilon$ later. Lemma 2 provides a set $F_{i}$ containing a constant fraction of the block-rows of $M^{(i)}$ so that $M_{\left[F_{i}, F_{i}\right]}^{(i)}$ is 4 -bDD. Theorem 4 then provides an operator that solves systems in $\boldsymbol{M}_{\left[F_{i}, F_{i}\right]}^{(i)}$ to $\epsilon$ accuracy in time $O\left(\log \epsilon^{-1}\right)$ times the number of nonzero entries in $\boldsymbol{M}_{\left[F_{i}, F_{i}\right]}^{(i)}$. This is at most the number of nonzero entries in $\boldsymbol{M}^{(i)}$, and thus at most $O\left(\left|C_{i}\right| / \epsilon^{2}\right)$. As each $F_{i}$ contains at least a constant fraction of the rows of $\boldsymbol{M}^{(i)}$, the depth of the recursion, $d$, is $O(\log n)$. Thus, we can obtain constant accuracy by setting $\epsilon=\Theta(1 / \log n)$. The time required to apply the resulting SCC would thus be $O\left(n \log ^{2} n \log \log n\right)$.

We can reduce the running time by setting $\epsilon_{1}$ to a constant and allowing it to shrink as $i$ increases. For example, setting $\epsilon_{i}=1 / 2(i+1)^{2}$ results in a linear time algorithm that produces a constant-factor approximation of the inverse of $\boldsymbol{M}$. We refine this idea in the next section.

\subsection{Linear Sized Approximate Inverses}

In this section we sketch the proof of Theorem 2 which tells us that every $b D D$ matrix has a linear-sized approximate inverse. The rest of the details appear in the full version $[12$, Section F].

The linear-sized approximate inverse of a $b D D$ matrix $M$ with $n$ block rows and columns is provided by a $3 / 4-$ approximate of the form $\boldsymbol{U}^{T} \boldsymbol{D} \boldsymbol{U}$ where $\boldsymbol{D}$ is block diagonal and $\boldsymbol{U}$ is block upper-triangular and has $O(n)$ nonzero blocks. As systems of linear equations in block-triangular matrices like $\boldsymbol{U}$ and $\boldsymbol{U}^{T}$ can be solved in time proportional to their number of nonzero blocks, this provides a linear time algorithm for computing a 3/4 approximation of the inverse of $\boldsymbol{M}$. By iteratively refining the solutions provided by the approximate inverse, this allows one to find $\epsilon$-accurate solutions to systems of equations in $M$ in time $O\left(m \log \epsilon^{-1}\right)$.

The matrix $\boldsymbol{U}$ that we construct, is sparse and block upper-triangular. Thus, solves in $\boldsymbol{U}$ and $\boldsymbol{U}^{T}$ can be performed with linear work and depth $O\left(\log ^{2} n\right)$. This results in a parallel algorithm for solving equations in $\boldsymbol{M}$ in work $O\left(m \log \epsilon^{-1}\right)$ and depth $O\left(\log ^{2} n \log \epsilon^{-1}\right)$. We remark that with some additional work (analogous to Section 7 of [13]), one could reduce this depth to $O\left(\log n \log \log n \log \epsilon^{-1}\right)$.

The key to constructing $\boldsymbol{U}$ is realizing that the algorithm JACOBI corresponds to multiplying by the matrix $\boldsymbol{Z}^{(k)}$ defined by the following equation,

$$
\boldsymbol{Z}^{(k)} \stackrel{\text { def }}{=} \sum_{i=0}^{k} \boldsymbol{X}_{[F, F]}^{-1}\left(-\boldsymbol{L}_{[F, F]} \boldsymbol{X}_{[F, F]}^{-1}\right)^{i} .
$$




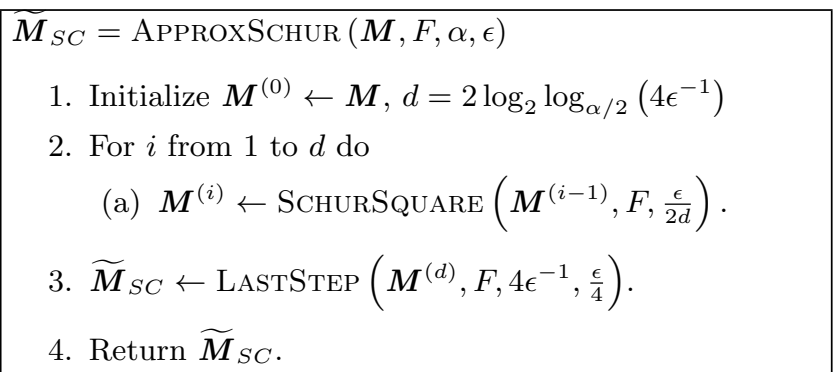

Figure 4: Pseudocode for Computing Spectral Vertex Sparsifiers

Moreover, this matrix is a polynomial in $\boldsymbol{X}$ and $\boldsymbol{L}$ of degree $\log (3 / \epsilon)$, where $\boldsymbol{M}_{\left[F_{i}, F_{i}\right]}^{(i)}=\boldsymbol{X}+\boldsymbol{L}$ where $\boldsymbol{L}$ is bDD, and $\boldsymbol{X}$ block diagonal such that $\boldsymbol{X} \succcurlyeq 2 \boldsymbol{L}$. To force $\boldsymbol{Z}^{k}$ to be a sparse matrix, we require that $\boldsymbol{L}$ be sparse.

If we use the algorithm BSDDSUBSET to choose $F_{i}$, then $\boldsymbol{L}$ need not be sparse. However, this problem is easily remedied by forbidding algorithm BSDDSUBSET from choosing any vertex of more than twice average degree. Thus, we can ensure that all $\boldsymbol{Z}^{(i)}$ are sparse.

Lemma 4. For every bDD matrix $M$ and every $\alpha \geq 0$, there is a subset $F$ of size at least $\frac{n}{16(1+\alpha)}$ such that $M_{[F, F]}$ is $\alpha-b D D$ and the number of nonzero blocks in each blockrow of $F$ at most twice the average number of nonzero blocks in a block-row of $\boldsymbol{M}$.

Proof. Discard every block-row of $\boldsymbol{M}$ that has more than twice the average number of nonzero blocks per rowblock. Then remove the corresponding row blocks. The remaining matrix has dimension at least $n / 2$. We can now use Lemma 2 to find an $\alpha$-bDD submatrix.

We obtain the $\boldsymbol{U}^{T} \boldsymbol{D} \boldsymbol{U}$ factorization by applying the inverse of the factorization (1):

$$
\begin{aligned}
& \boldsymbol{M =}\left[\begin{array}{cc}
\boldsymbol{I} & 0 \\
\boldsymbol{M}_{[F, F]}^{-1} \boldsymbol{M}_{[F, C]} & \boldsymbol{I}
\end{array}\right]\left[\begin{array}{cc}
\boldsymbol{M}_{[F, F]} & 0 \\
0 & S c(\boldsymbol{M}, F)
\end{array}\right] \\
& {\left[\begin{array}{cc}
\boldsymbol{I} & \boldsymbol{M}_{[C, F]} \boldsymbol{M}_{[F, F]}^{-1} \\
0 & \boldsymbol{I}
\end{array}\right] . }
\end{aligned}
$$

In the left and right triangular matrices we replace $\boldsymbol{M}_{[F, F]}^{-1}$ with the polynomial we obtain from JACOBI. In the middle matrix, it suffices to approximate $\boldsymbol{M}_{[F, F]}$ by a block diagonal matrix, and $S c(\boldsymbol{M}, F)$ by a factorization of its sparse approximation given by Theorem 3 . The details, along with a careful setting of $\epsilon_{i}$, are carried out in the full version 12 , Section F].

\subsection{Spectral Vertex Sparsification Algorithm}

In this section, we outline a procedure APPROxScHur that efficiently approximates the Schur complement of a bDD matrix $\boldsymbol{M}$ w.r.t. a set of indices $F$ s.t. $\boldsymbol{M}_{[F, F]}$ is $\alpha$-bDD. The procedure ApproxSchur is described in figure 4 The following lemma summarizes its guarantees.

Lemma 5. Let $M$ be a bDD matrix with index set $V$, and $m$ nonzero blocks. Let $F \subseteq V$ be such that $M_{[F, F]}$ is $\alpha-b D D$ for some $\alpha \geq 4$. The algorithm $\operatorname{Approx} \operatorname{Schur}(M, F, \epsilon)$, returns a matrix $\widetilde{\boldsymbol{M}}_{S C}$ s.t.
1. $\widetilde{\boldsymbol{M}}_{S C}$ has $O\left(m\left(\epsilon^{-1} \log \log \epsilon^{-1}\right)^{O\left(\log \log \epsilon^{-1}\right)}\right)$ nonzero blocks, and

2. $\widetilde{M}_{S C} \approx_{\epsilon} S c(M, F)$,

in $O\left(m\left(\epsilon^{-1} \log \log \epsilon^{-1}\right)^{O\left(\log \log \epsilon^{-1}\right)}\right)$ work and $O\left(\log n\left(\log \log \epsilon^{-1}\right)\right)$ depth.

In this section, we sketch the procedures SCHURSQUARE and LAstStep, and a proof of the above lemma. A complete proof and descriptions of the procedures are given in the full version [12, Section G].

First consider a very simple special case: where $F$ is a singleton, $F=\{i\}$. Let $C=V \backslash F$ be the remaining indices.

$$
S c(M, i)=M_{[C, C]}-M_{[C, i]} M_{[i, i]}^{-1} M_{[i, C]}
$$

Thus, if $\boldsymbol{M}_{[C, i]}$ has $k$ nonzero blocks, $S c(\boldsymbol{M}, i)$ could have $k^{2}$ additional nonzero blocks compared to $\boldsymbol{M}_{[C, C]}$, potentially making it dense. If $\boldsymbol{M}$ were a graph Laplacian, then $\boldsymbol{M}_{[C, i]} \boldsymbol{M}_{[i, i]}^{-1} \boldsymbol{M}_{[i, C]}$ would represent the adjacency matrix of a weighted clique. In the full version [12, Section J], we construct weighted expanders that allow us to $\epsilon$-approximate $S c(M, i)$ in this case using $m+O\left(k \epsilon^{-4}\right)$ edges. In Section G.4 [12], we show how to use such weighted expanders to sparsify $S c(\boldsymbol{M}, i)$ when $\boldsymbol{M}$ is a bDD matrix.

This reduction can also be performed in parallel. If $F$ is such that $\boldsymbol{M}_{[F, F]}$ is block diagonal, we can approximate $S c(M, F)$ by expressing $\boldsymbol{M}_{[C, i]} \boldsymbol{M}_{[i, i]}^{-1} \boldsymbol{M}_{[i, C]}$ as

$$
\sum_{i \in F} \boldsymbol{M}_{[C, i]} \boldsymbol{M}_{[i, i]}^{-1} \boldsymbol{M}_{[i, C]}
$$

and using $|F|$ weighted expanders. However, $\boldsymbol{M}_{[F, F]}$ may not be diagonal. Instead, we give a procedure SCHURSQUARE that generates $\boldsymbol{M}^{\prime}$ that is better approximated by its diagonal.

Invoking SchurSquARE a few times leads a sequence of matrices $\boldsymbol{M}_{[F, F]}^{(0)}, \boldsymbol{M}_{[F, F]}^{(1)}, \ldots, \boldsymbol{M}_{[F, F]}^{(i)}$. We will show that $\boldsymbol{M}_{[F, F]}^{(i)}$ is $\epsilon$-approximated by its diagonal and we call the procedure LAstSteP to approximate $S c\left(M^{(i)}, F\right)$. An additional caveat is that replacing $\boldsymbol{M}_{[F, F]}^{(i)}$ by its diagonal at this step gives errors that are difficult to bound. We discuss the correct approximation below

SCHURSQUARE is based on a squaring identity for matrix inverse developed in 18. Given a splitting of $\boldsymbol{M}_{[F, F]}$ into $\boldsymbol{D}-\boldsymbol{A}$, where $\boldsymbol{D}$ is block-diagonal, and $\boldsymbol{A}$ has its diagonal blocks as zero, it relies on the fact that the matrix $M_{2}$ defined in Equation (3) satisfies $S c(\boldsymbol{M}, F)=S c\left(\boldsymbol{M}_{2}, F\right)$. Furthermore, we can show that if $\boldsymbol{M}$ is $\alpha$-bDD, $\boldsymbol{D}-\boldsymbol{A} \boldsymbol{D}^{-1} \boldsymbol{A}$ is $\alpha^{2}$-bDD, which indicates that the block on $[F, F]$ rapidly approaches being diagonal.

As $M_{2}$ may be dense, we construct a sparse approximation to it. Since $\boldsymbol{D}$ is diagonal, we can construct sparse approximations to the blocks $\left(\boldsymbol{M}_{2}\right)_{[F, F]}$ and $\left(\boldsymbol{M}_{2}\right)_{[C, C]}$ in a manner analogous to the case of diagonal $\boldsymbol{M}_{[F, F]}$. Similarly, we use bipartite expanders to construct sparse approximations to $\left(\boldsymbol{M}_{2}\right)_{[C, F]}$ and $\left(\boldsymbol{M}_{2}\right)_{[F, C]}$.

Our sequence of calls to SchurSquARE terminates with $\boldsymbol{M}_{[F, F]}^{(i)}$ being roughly $\epsilon^{-1}$-bDD. We then return $\operatorname{LastStep}\left(\boldsymbol{M}^{(i)}, F, \epsilon^{-1}, \epsilon\right)$. As mentioned above, we cannot just replace $\boldsymbol{M}_{[F, F]}^{(i)}$ by its diagonal. Instead, LASTSTEP performs one step of squaring similar to SCHURSQUARE with a 


$$
\boldsymbol{M}_{2}=\frac{1}{2}\left[\begin{array}{cc}
\boldsymbol{D}-\boldsymbol{A} \boldsymbol{D}^{-1} \boldsymbol{A} & \boldsymbol{M}_{[F, C]}+\boldsymbol{A} \boldsymbol{D}^{-1} \boldsymbol{M}_{[F, C]} \\
\boldsymbol{M}_{[C, F]}+\boldsymbol{M}_{[C, F]} \boldsymbol{D}^{-1} \boldsymbol{A} & 2 \boldsymbol{M}_{[C, C]}-\boldsymbol{M}_{[C, F]} \boldsymbol{D}^{-1} \boldsymbol{M}_{[F, C]}
\end{array}\right]
$$

key difference: Rather than expressing $\boldsymbol{M}_{[F, F]}^{(i)}$ as $\boldsymbol{D}-\boldsymbol{A}$, it expresses it as $\boldsymbol{X}+\boldsymbol{L}$, where $\boldsymbol{X}$ is block-diagonal, and $\boldsymbol{L}$ is just barely bDD. With this splitting, it constructs $\boldsymbol{M}^{(\text {last })}$ after performing one iteration similar to Eq. (3). After this step, it replaces the $\boldsymbol{M}_{[F, F]}^{(\text {last })}$ block with the block-diagonal matrix $\boldsymbol{X}$. Again, we directly produce sparse approximations to $\boldsymbol{M}^{\text {(last) }}$ and its Schur complement via weighted (bipartite) expanders. A precise description and proofs are given in the full version [12, Section G.2].

\subsection{Sparsifying bDD Matrices}

The main technical hurdle left to address is how we sparsify bDD matrices. We to do this both to approximate $\boldsymbol{M}$ by $\boldsymbol{M}^{(0)}$, if $\boldsymbol{M}$ is not already sparse, and to ensure that all the matrices $\boldsymbol{M}^{(i)}$ remain sparse. While the spectral vertex sparsification algorithm described in the previous section allows us to compute an approximation to a Schur complement $S c\left(\boldsymbol{M}^{(i)}, F_{i}\right)$, it is sparse only when $\boldsymbol{M}^{(i)}$ is already sparse. As we iteratively apply this procedure, the density of the matrices produced will grow unacceptably. We overcome this problem by occasionally applying another sparsification routine that substantially decreases the number of nonzero blocks. The cost of this sparsification routine is that it requires solving systems of equations in sparse $b D D$ matrices. We, of course, do this recursively.

Our sparsification procedure begins by generalizing the observation that graph Laplacians can be sparsified by sampling edges with probabilities determined by their effective resistances 22]. We define a block analog of leverage scores that provides probabilities of sampling blocks so that the resulting sampled matrix approximates the original and has $O(n \log n)$ nonzero blocks with high probability. To compute this block analog of leverage scores we employ a recently introduced procedure of Cohen et. al. 6.

Once we generalize their results to block matrices, they show that we can obtain sufficiently good estimates of the block leverage scores by computing leverage scores in a bDD matrix obtained by randomly subsampling blocks of the original. The block leverage scores in this matrix are obtained by solving a logarithmic number of linear equations in this subsampled matrix. Thus, our sparsification procedure requires constructing a solver for a subsampled matrix and then applying that solver a logarithmic number of times. We compute this solver recursively.

There is a tradeoff between the number of nonzero blocks in the subsampled system and in the resulting approximation of the original matrix. If the original matrix has $m$ nonzero blocks and we subsample to a system of $m / K$ nonzero blocks, then we obtain an $\epsilon$-approximation of the original matrix with $O\left(K \epsilon^{-2} n \log n\right)$ nonzero blocks.

The details of the analysis of the undersampling procedure appear in the full version 12 , Section H].

\subsection{The Main Algorithm}

We now explain how we prove Theorem 1 1 The details supporting this exposition appear in the full version 12 ,
Section I]. Our main goal is to control the density of the Schur complement chain as we repeatedly invoke Lemma 5

Starting from some $\boldsymbol{M}^{(0)}$, we compute sets $F_{i}$ (via calls to BDDSUBSET), approximate solvers $\boldsymbol{Z}^{(i)}$ (via JACOBI), and approximations of Schur complements $\boldsymbol{M}^{(i)}$ (via ApProxSCHUR), until we obtain a matrix $\boldsymbol{M}^{(i)}$ such that its dimension is a smaller than that of $\boldsymbol{M}^{(0)}$ by a large constant factor (like 4). While the dimension of $\boldsymbol{M}^{(i)}$ is much smaller, its number of nonzero blocks is potentially larger by an even larger factor. We use the procedure described in the previous section to sparsify it. This sparsification procedure produces a sparse approximation of the matrix at the cost of solving systems of equations in a subsampled version of that matrix. Some care is required to balance the cost of the resulting recursion.

We now sketch an analysis of a nearly linear time algorithm that results from a simple choice of parameters. We optimize the parameter choice and analysis in the full version [12. Section I]. Let $n$ be the dimension of $\boldsymbol{M}^{(0)}$ and let $m$ be its number of nonzero blocks. To begin, assume that $m \leq n \Delta \log ^{3} n$, for a $\Delta$ to be specified later. We call this the sparse case, and address the case of dense $\boldsymbol{M}$ later.

We consider fixing $\epsilon_{i}=c / \log n$ for all $i$, for some constant c. As the depth of the Schur complement chain is $O(\log n)$, this results in a solver with constant accuracy. A constant number of iterations of the procedure described above are required to produce an $\boldsymbol{M}^{(i)}$ whose dimension is a factor of 4 smaller than $\boldsymbol{M}^{(0)}$. Lemma 5 tells us that the edge density of this $\boldsymbol{M}^{(i)}$ is potentially higher than that of $\boldsymbol{M}^{(0)}$ by a factor of

$$
\begin{aligned}
O\left(\left(\epsilon^{-1} \log \log \epsilon^{-1}\right)^{O\left(\log \log \epsilon^{-1}\right)}\right) & =(\log n)^{O(\log \log \log n)} \\
& =n^{o(1)}
\end{aligned}
$$

Set $\Delta$ to be this factor. We use the sparsification procedure from the previous section to guarantee that no matrix in the chain has density higher than that of this matrix, which is upper bounded by $(m / n) \Delta=\Delta^{2} \log ^{3} n$.

Setting $K=2 \Delta$, the subsampling produces a matrix of density half that of $\boldsymbol{M}^{(0)}$, and it produces a sparse approximation of $\boldsymbol{M}^{(i)}$ of density $O\left(K \epsilon_{i}^{-2} \log n\right) \leq O\left(\Delta \log ^{3} n\right)$, which, by setting constants appropriately, we can also force to be half that of $\boldsymbol{M}^{(0)}$. In order to perform the sparsification procedure, we need to construct a Schur complement chain for the subsampled matrix, and then use it to solve $O(\log n)$ systems of linear equations. The cost of using this chain to solve equations in the subsampled system is at most $O\left(n \Delta^{2} \log ^{4} n\right)$, and the cost of using the solutions to these equations to sparsify $\boldsymbol{M}^{(i)}$ is $O(m \log n)$. The cost of the calls to BDDSUBSET and APPROXSCHUR are proportional to the number of edges in the matrices, which is $O\left(n \Delta \log ^{4} n\right)$.

We repeat this procedure all the way down the chain, only using sparsification when the dimension of $\boldsymbol{M}^{(i)}$ shrinks by a factor of 4 . Since none of the matrices that we generate have density higher than $\Delta \log ^{3} n$, we remain in the sparse case. Let $T_{\text {sparse }}(n)$ be the time required to construct a solver chain on systems of size $n$ with $m \leq n \Delta \log ^{3} n$. 
We obtain the following recurrence

$$
\begin{aligned}
T_{\text {sparse }}(n) & \leq 2 T_{\text {sparse }}(n / 4)+n \Delta^{2} \log ^{4} n+m \log n+m \Delta \\
& \leq 2 T_{\text {sparse }}(n / 4)+n \Delta^{2} \log ^{4} n+n \Delta \log n+n \Delta^{2},
\end{aligned}
$$

which gives

$$
T_{\text {sparse }}(n) \leq O\left(n \Delta^{2}+n \Delta^{2} \log ^{4} n\right) \leq n^{1+o(1)} .
$$

To handle the case of dense $\boldsymbol{M}$, we repeatedly sparsify while keeping $n$ fixed until we obtain a matrix with fewer than $n \Delta \log ^{3} n$ edges, at which point we switch to the algorithm described above. The running time of this algorithm on a graph with $m$ edges, $T_{\text {dense }}(m)$, satisfies the recurrence

$T_{\text {dense }}(m) \leq\left\{\begin{array}{lc}T_{\text {sparse }}(n) & \text { if } m \leq n \Delta \log ^{3} n, \text { and } \\ 2 T_{\text {dense }}(m / 2)+n \Delta^{2} \log ^{4} n+m \log n+m \Delta & \text { otherwise. }\end{array}\right.$

Thus $T_{\text {dense }}(m)$ is upper bounded bounded by $O\left(m n^{o(1)}+\right.$ $\left.n^{1+o(1)}\right)$.

We tighten this bound in the full version 12 , Section I] to prove Theorem 1 by carefully choosing the parameters to accompany a sequence $\boldsymbol{\epsilon}$ that starts constant and decreases slowly.

\section{SUMMARY}

We introduce a new approach to solving systems of linear equations that gives the first nearly linear time algorithms for solving systems in connection Laplacians and the first proof that connection Laplacians have linear-sized approximate inverses. This was unknown even for graph Laplacians.

Our algorithms build on ideas introduced in 18. and are a break from those used in the previous work on solving systems of equations in graph Laplacians $26,24,9,10,8$, 5]. Those algorithms all rest on support theory 4, originally introduced by Vaidya 26], and rely on the fact that the Laplacian of one edge is approximated by the Laplacian of a path between its endpoints. No analogous fact is true for connection Laplacians, even those with complex entries for $r=1$.

Instead, our algorithms rely on many new ideas, the first being that of sparsifying the matrices that appear during elimination. Other critical ideas are finding $\alpha$-bDD subsets of vertices to eliminate in bulk, approximating Schur complements without computing them explicitly, and the use of sub-sampling to sparsify in a recursive fashion. To efficiently compute approximations of the Schur complements, we introduce a new operation that transforms a matrix into one with the same Schur complement but a much better conditioned upper block (3). To obtain the sharp bounds in Theorem 1. we exploit a new linear-time algorithm for constructing linear-sized sparse approximations to implicitly represented weighted cliques whose edge weights are products of weights at vertices (see full version $[12$, Section J]), and extend this to the analog for bDD matrices (see full version [12, Section G.4]).

\section{REFERENCES}

[1] B. Alexeev, A. S. Bandeira, M. Fickus, and D. G. Mixon. Phase retrieval with polarization. SIAM Journal on Imaging Sciences, 7(1):35-66, 2014.
[2] M. Arie-Nachimson, S. Z. Kovalsky, I. Kemelmacher-Shlizerman, A. Singer, and R. Basri. Global motion estimation from point matches. In $3 D$ Imaging, Modeling, Processing, Visualization and Transmission (3DIMPVT), 2012 Second International Conference on, pages 81-88. IEEE, 2012.

[3] A. S. Bandeira, A. Singer, and D. A. Spielman. A cheeger inequality for the graph connection laplacian. SIAM Journal on Matrix Analysis and Applications, 34(4):1611-1630, 2013.

[4] M. Bern, J. Gilbert, B. Hendrickson, N. Nguyen, and S. Toledo. Support-graph preconditioners. SIAM J. Matrix Anal. \&f Appl, 27(4):930-951, 2006.

[5] M. B. Cohen, R. Kyng, G. L. Miller, J. W. Pachocki, R. Peng, A. B. Rao, and S. C. Xu. Solving sdd linear systems in nearly mlog $1 / 2 \mathrm{n}$ time. In Proceedings of the 46th Annual ACM Symposium on Theory of Computing, STOC '14, pages 343-352, New York, NY, USA, 2014. ACM.

[6] M. B. Cohen, Y. T. Lee, C. Musco, C. Musco, R. Peng, and A. Sidford. Uniform sampling for matrix approximation. arXiv preprint arXiv:1408.5099, 2014.

[7] M. K. de Carli Silva, N. J. A. Harvey, and C. M. Sato. Sparse sums of positive semidefinite matrices. CoRR, abs/1107.0088, 2011.

[8] J. A. Kelner, L. Orecchia, A. Sidford, and Z. A. Zhu. A simple, combinatorial algorithm for solving sdd systems in nearly-linear time. In Proceedings of the 45th annual ACM symposium on Symposium on theory of computing, pages 911-920. ACM, 2013.

[9] I. Koutis, G. Miller, and R. Peng. Approaching optimality for solving SDD linear systems. In Foundations of Computer Science (FOCS), 2010 51st Annual IEEE Symposium on, pages 235-244, 2010.

[10] I. Koutis, G. Miller, and R. Peng. A nearly- $m \log n$ time solver for SDD linear systems. In Foundations of Computer Science (FOCS), 2011 52nd Annual IEEE Symposium on, pages 590-598, 2011.

[11] D. Krishnan, R. Fattal, and R. Szeliski. Efficient preconditioning of laplacian matrices for computer graphics. ACM Transactions on Graphics (TOG), 32(4):142, 2013.

[12] R. Kyng, Y. T. Lee, R. Peng, S. Sachdeva, and D. A. Spielman. Sparsified cholesky and multigrid solvers for connection laplacians. CoRR, abs/1512.01892, 2015.

[13] Y. T. Lee, R. Peng, and D. A. Spielman. Sparsified cholesky solvers for SDD linear systems. CoRR, abs/1506.08204, 2015.

[14] S. Marchesini, Y.-C. Tu, and H.-t. Wu. Alternating projection, ptychographic imaging and phase synchronization. arXiv preprint arXiv:1402.0550, 2014.

[15] J. A. Meijerink and H. A. v. d. Vorst. An iterative solution method for linear systems of which the coefficient matrix is a symmetric $m$-matrix. Mathematics of Computation, 31(137):148-162, 1977.

[16] L. Orecchia and N. K. Vishnoi. Towards an sdp-based approach to spectral methods: a nearly-linear-time algorithm for graph partitioning and decomposition. In Proceedings of the Twenty-Second Annual ACM-SIAM Symposium on Discrete Algorithms, SODA '11, pages 532-545. SIAM, 2011. 
[17] O. OİĹzyesìğil, A. Singer, and R. Basri. Stable camera motion estimation using convex programming. SIAM Journal on Imaging Sciences, 8(2):1220-1262, 2015.

[18] R. Peng and D. A. Spielman. An efficient parallel solver for SDD linear systems. In Symposium on Theory of Computing, STOC 2014, New York, NY, USA, May 31 - June 03, 2014, pages 333-342, 2014.

[19] Y. Shkolnisky and A. Singer. Viewing direction estimation in cryo-em using synchronization. SIAM journal on imaging sciences, 5(3):1088-1110, 2012.

[20] A. Singer and Y. Shkolnisky. Three-dimensional structure determination from common lines in cryo-em by eigenvectors and semidefinite programming. SIAM journal on imaging sciences, 4(2):543-572, 2011.

[21] A. Singer and H.-T. Wu. Vector diffusion maps and the connection laplacian. Communications on pure and applied mathematics, 65(8), 2012.

[22] D. Spielman and N. Srivastava. Graph sparsification by effective resistances. SIAM Journal on Computing, 40(6):1913-1926, 2011.
[23] D. A. Spielman and S.-H. Teng. A local clustering algorithm for massive graphs and its application to nearly linear time graph partitioning. SIAM Journal on Computing, 42(1):1-26, 2013.

[24] D. A. Spielman and S.-H. Teng. Nearly-linear time algorithms for preconditioning and solving symmetric, diagonally dominant linear systems. SIAM. J. Matrix Anal. É Appl., 35:835âĂŞ885, 2014.

[25] U. Trottenberg, C. W. Oosterlee, and A. Schuller. Multigrid. Academic press, 2000.

[26] P. M. Vaidya. Solving linear equations with symmetric diagonally dominant matrices by constructing good preconditioners. Unpublished manuscript UIUC 1990 A talk based on the manuscript was presented at the IMA Workshop on Graph Theory and Sparse Matrix Computation, October 1991, Minneapolis., 1990.

[27] Z. Zhao and A. Singer. Rotationally invariant image representation for viewing direction classification in cryo-em. Journal of structural biology, 186(1):153-166, 2014 . 